\title{
U.S. Multicenter Pilot Study of Daily Consensus Interferon (CIFN) Plus Ribavirin for "Difficult-to-Treat" HCV Genotype 1 Patients
}

\author{
Samuel B. Ho • Bashar Aqel $\cdot$ Eric Dieperink $\cdot$ Shanglei Liu • \\ Lori Tetrick · Yngve Falck-Ytter · Charles DeComarmond • \\ Coleman I. Smith · Daniel P. McKee - William Boyd • \\ Clark C. Kulig $\cdot$ Edmund J. Bini $\cdot$ Marcos C. Pedrosa
}

Received: 9 February 2010/Accepted: 17 November 2010/Published online: 11 January 2011

(C) The Author(s) 2011. This article is published with open access at Springerlink.com

\begin{abstract}
Background Patients with chronic hepatitis C genotype 1 $(\mathrm{HCV}-1)$ and difficult-to-treat characteristics respond poorly to pegylated interferon alfa and ribavirin (RBV), and could benefit from an interferon with increased activity (consensus interferon or CIFN), favorable viral kinetics from daily dosing, and a longer duration of therapy. The purpose of this pilot study was to determine the efficacy and safety of daily CIFN + RBV for initial treatment of patients with HCV-1 infection.

Methods Patients with difficult-to-treat characteristics (92\% male, 33\% African American, 78\% Veterans Affairs [VA]; 67\% high viral load, 59\% stage 3-4 fibrosis, and mean weight of $204 \mathrm{lbs}$ ) were enrolled at seven VA and two community medical centers. They were randomized to daily CIFN (15 mcg/day SQ) and RBV (1-1.2 g/d PO)
\end{abstract}

S. B. Ho $(\bowtie) \cdot$ S. Liu

VA San Diego Healthcare System, 3350 La Jolla Village Drive, San Diego, CA 92161, USA

e-mail: samuel.ho2@va.gov

S. B. Ho

Department of Medicine, University of California, San Diego, San Diego, CA, USA

B. Aqel

Department of Medicine, Minneapolis VA Medical Center,

Minneapolis, MN, USA

E. Dieperink · L. Tetrick

Departments of Psychiatry and Research, Minneapolis VA

Medical Center, Minneapolis, MN, USA

Y. Falck-Ytter

Louis Stokes Cleveland VA Medical Center, Cleveland, OH, USA given for either 52 weeks (group $\mathrm{A}, n=33$ ) or 52-72 weeks (from time of viral response +48 weeks) (group $\mathrm{B}, n=31$ ).

Results Intention to treat analysis for treatment groups $\mathrm{A}$ and B demonstrated 33\% (11/33) and 32\% (10/31) sustained virologic response (SVR), respectively. Only 2/31 patients in group B received more than 52 weeks of treatment. The overall group demonstrated a $31 \%$ (20/64) rapid virologic response rate (RVR), 54\% (34/64) end of treatment virologic response and a 33\% (21/64) SVR. Patients with RVR at 4 weeks, early virologic response from 8-12 weeks, and late virologic response from 16-24 weeks demonstrated SVR of 75\% (15/20), $31 \%$ (4/13), and 22\% (2/9), respectively. Overall early non-protocol discontinuation occurred in 26/64 (40\%) patients.

\author{
C. DeComarmond \\ W. G. Hefner VA Medical Center, Salisbury, NC, USA \\ C. I. Smith \\ Minnesota Gastroenterology, Minneapolis, MN, USA \\ D. P. McKee \\ Northland Gastroenterology, Duluth, MN, USA \\ W. Boyd \\ James A. Haley Veterans' Hospital, Tampa, FL, USA \\ C. C. Kulig \\ VA Eastern Colorado Healthcare System, Denver, CO, USA \\ E. J. Bini \\ VA New York Harbor Healthcare System, New York, NY, USA \\ M. C. Pedrosa \\ VA Boston Healthcare System, Boston, MA, USA
}


Conclusion Daily CIFN and ribavirin for initial treatment of HCV-1 patients has potential for achieving a relatively high RVR rate, but discontinuations are frequent and successful use of this regimen is highly dependent on adequate patient support to maintain adherence.

Keywords Consensus interferon - Hepatitis C . Ribavirin - Veterans Affairs

\section{Introduction}

Hepatitis $\mathrm{C}$ virus (HCV) is a leading cause of chronic liver disease in the United States, often resulting in cirrhosis, liver failure, or hepatocellular carcinoma in infected individuals [1]. The projected mortality due to HCV-related liver disease and hepatocellular carcinoma is expected to total over 200,000 individuals between 2010 and 2019 in the United States [2]. Current treatment for hepatitis C largely consists of pegylated interferon alfa 2 a plus ribavirin or pegylated interferon alfa $2 b$ plus ribavirin [3]. This treatment is inadequate for many patients with $\mathrm{HCV}$ genotype 1 , since the majority of these patients studied in registration trials do not respond (termed non-responders) or respond but relapse (termed relapsers) following termination of treatment [4-7]. Characteristics that are associated with a worse response to treatment include genotype 1 infection and high viral blood levels, increased body mass index, African American ethnicity, male gender, and the presence of advanced liver fibrosis. Data from a multicenter Veterans Affairs (VA) study indicate that a higher percentage of patients in the VA system have these characteristics, which may be responsible for poor treatment response rates [8]. A large retrospective review of SVR rates in veterans with chronic hepatitis $\mathrm{C}$ indicated that SVR was attained in only $20 \%$ of patients with treatment naïve genotype 1 patients treated with pegylated interferons and ribavirin [9].

Consensus interferon (CIFN), also known as interferon alfacon-1 or Infergen, is a non-naturally occurring type-1 interferon that was derived by scanning the sequences of several nonallelic natural interferon alpha subtypes and assigning the most frequently observed amino acid in each corresponding position, producing a consensus sequence. CIFN differs from interferon alfa-2a and interferon alfa- $2 b$ by 19 and 18 amino acids, respectively. CIFN has been shown to have greater biologic activity relative to other interferons, which may be explained by the fact that CIFN binds with higher affinity to the interferon receptor complex, producing greater signaling output and increased production of interferon stimulated genes [10-13].

Several clinical studies have suggested that CIFN may have greater antiviral activity in both treatment naïve and prior treatment nonresponder patients with genotype 1 hepatitis $\mathrm{C}$ infection, particularly if given as a daily injection [14-18]. These data suggested that a strategy using daily CIFN may be more effective for genotype 1 HCV patients than currently available treatments with pegylated interferons and ribavirin. Emerging data also suggest that patients who have a "late" early virologic response, meaning that they have a virologic response (defined as a negative HCV PCR test) on pegylated interferon and ribavirin therapy between 12 and 24 weeks, will have an improved sustained response rate if they are treated for up to 72 weeks compared with 48 weeks [19, 20]. These data suggest the hypothesis that patients who have a "late" early virologic response with CIFN and ribavirin between 12 and 24 weeks may also benefit from a course of therapy that is extended to up to 72 weeks in duration.

Therefore, the goals of this pilot study are to determine the tolerability and efficacy of daily CIFN plus ribavirin when given for 52 weeks or an extended duration of therapy. The target population was recruited from centers with higher percentage of patients with difficult-to-treat characteristics, which include the following characteristics: genotype 1, a North American patient population, predominantly male gender, and VA location.

\section{Methods}

\section{Study protocol}

This is an investigator-initiated randomized, open label, multicenter, U.S.-based trial. The primary objective of the study was to provide pilot data on the efficacy and tolerability of daily CIFN and ribavirin given for 52 weeks in patients with chronic hepatitis $\mathrm{C}$ genotype 1 . The trial was designed to study genotype 1 patients with difficult-to-treat characteristics, and included primarily male patients at VA medical centers. The secondary objective was to obtain pilot data to determine the feasibility of treating patients for longer than 52 weeks based on the time of initial viral response during the first 24 weeks. All patients gave informed consent and the study was approved by the institutional review boards of all study sites. The study was registered with ClinicalTrials.gov identifier NCT00211692.

All patients that enrolled were randomized at a 1:1 ratio to daily CIFN (15 mcg day ${ }^{-1} \mathrm{SQ}$ ) and ribavirin (1-1.2 g day $^{-1} \mathrm{PO}$ ) given for up to 52 weeks (group A) or daily CIFN $\left(15 \mathrm{mcg} \mathrm{d}^{-1} \mathrm{SQ}\right)$ and ribavirin (1-1.2 $\left.\mathrm{g} \mathrm{day}^{-1} \mathrm{PO}\right)$ given from 52 to 72 weeks (group B) depending on the time to achieving viral negativity (Fig. 1).

The presence of $\mathrm{HCV}$ in serum was determined by several HCV quantitative tests from week 4 through week 20. The type of assay varied between sites and changed 
Fig. 1 Study design and patient outcome

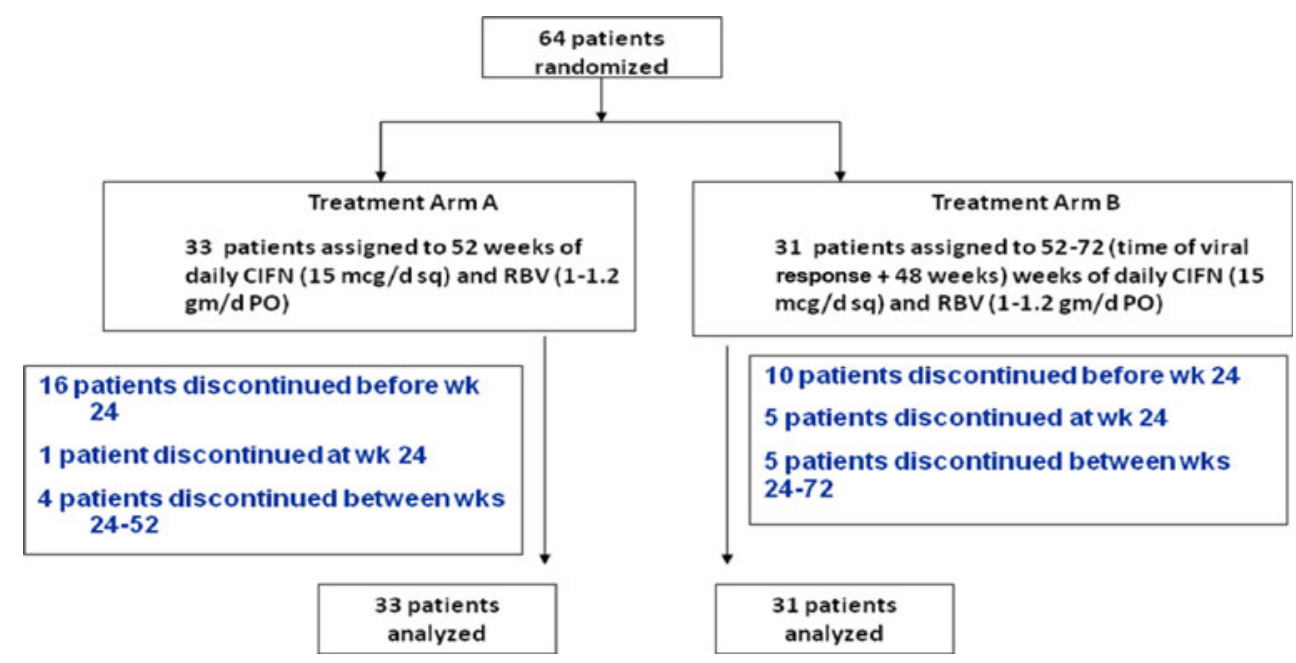

over the course of the study from 2005 to 2008. Assays used included the AMPLICOR HCV MONITOR Test v2.0, Roche Diagnostics, Pleasanton, CA (lower limit of detection $600 \mathrm{IU} \mathrm{ml}^{-1}$ ); $\mathrm{COBAS}^{\circledR}$ TaqMan ${ }^{\circledR} \mathrm{HCV}$ Test, lower limit of detection 25-50 IU ml $^{-1}$, Roche Diagnostics, Pleasanton, CA; and Abbott Celera HCV Quantification ASR Assay, Abbott Park, IL (lower limit of $25 \mathrm{IU} \mathrm{ml}^{-1}$ ). We defined a negative quantitative test as $<600 \mathrm{IU} \mathrm{ml}^{-1}$ for the purposes of this study. Subsequent HCV levels at week 24, end of treatment, and at 24 weeks after the end of treatment were determined using a qualitative HCV polymerase chain reaction $(\mathrm{PCR})$ test $\left(\mathrm{COBAS}^{\circledR}\right.$ AMPLICOR HCV Test, v2.0, and AMPLICOR ${ }^{\circledR}$ HCV Test, v2.0, Roche Diagnostics, Pleasanton, CA), with at least a lower limit of detection of 50-100 IU ml ${ }^{-1}$. Viral negativity through week 20 was defined as below the lower limit of detection with a quantitative test of at least $<600 \mathrm{IU} \mathrm{ml}^{-1}$; and viral negativity at week 24, end of treatment, and end of followup was defined as below the lower detection limit of the qualitative assay of at least $<100 \mathrm{IU} \mathrm{ml}^{-1}$. A rapid virologic response (RVR) is defined as viral negativity at week 4; an early virologic response is defined as $\geq 2-\log$ decrease in viremia or viral negativity at week 12; and an end of treatment response (ETR) is defined as viral negativity at the end of the treatment. A late virologic response (LVR) is defined as viral negativity occurring between week 12 and week 24. A sustained virologic response (SVR) is defined as a negative qualitative HCV PCR test taken 24 weeks after the end of CIFN/ribavirin therapy.

For patients in group $\mathrm{A}$, if there was a $\geq 2-\log$ drop in viremia at week 12 the therapy was continued. If the patient had a negative qualitative PCR test for HCV RNA at week 24 they continued to be treated for a total of 52 weeks. The treatment was discontinued if they did not have a $\geq 2-\log$ drop at week 12 or if they did not become negative for HCV RNA by qualitative PCR by 24 weeks on therapy.
For patients in group B, early virologic response was determined every 4 weeks to week 20 and 24, until a virologic response was demonstrated (defined as a $\geq 2-\log$ drop in viral levels from baseline or undetectable viral load) by quantitative PCR. Once patients attained a virologic response by quantitative PCR their treatment was continued for an additional 48 weeks. (For example, for patients who had less than a two log drop at week 4 , but attained $\mathrm{a} \geq 2-\log$ drop in viral levels by quantitative PCR at week 8 , the recommended duration of treatment was 8 weeks +48 weeks $=56$ weeks. Similarly, if a patient attained a virologic response only by week 20 , then the recommended duration of treatment was 20 weeks + 48 weeks $=68$ weeks. Also, if patients did not attain a 2-log drop by week 20 but had a negative qualitative PCR at 24 weeks, then total recommended treatment duration was $24+48$ weeks $=72$ weeks). The minimal duration of 52 weeks was chosen because this provided 48 weeks of treatment beyond the first viral negative point at 4 weeks. Again as in group A, treatment was discontinued if patients did not become negative for HCV RNA by qualitative PCR by 24 weeks on therapy.

Patients were required to have active HCV genotype 1 infection and have no obvious contraindications for antiviral treatment, documentation of the presence of circulating hepatitis $\mathrm{C}$ virus by a positive hepatitis C PCR test, a positive $\mathrm{HCV}$ genotype test for genotype 1, and a liver biopsy (within the previous 5 years) that is compatible with chronic hepatitis. Patients were between the ages of 18 and 65 years. Patients were enrolled according to current community standards of care and individual physician judgment and there were no specific exclusions for preexisting psychiatric or substance abuse co-morbidities. Female patients of childbearing age were required to have a negative pregnancy test at entry within $48 \mathrm{~h}$ of starting treatment and practice contraception. Patients were required to have compensated liver disease with the 
following laboratory results at entry: hemoglobin $\geq 12 \mathrm{~g}$ $\mathrm{dl}^{-1}$ for females and $\geq 13 \mathrm{~g} \mathrm{dl}^{-1}$ for males, white blood count $\geq 2,000 \mathrm{~mm}^{-3}$, neutrophil count $\geq 1,500 \mathrm{~mm}^{-3}$, platelets $\geq 75,000 \mathrm{~mm}^{-3}$, albumin $\geq 3.0 \mathrm{~g} \mathrm{dl}^{-1}$, total bilirubin $<2.0$, serum creatinine $\leq 1.4 \mathrm{mg} \mathrm{dl}^{-1}$, INR $\leq 1.8$, and TSH within normal limits.

Growth factors were allowed in this study as per current standard practice, but only after and in the context of the dose reduction schedule for CIFN and ribavirin. Step-wise dose reductions for changes in hemoglobin, platelets, and white blood count was performed as appropriate and as indicated by the protocol. Patients who developed anemia defined as $\mathrm{Hg}<10 \mathrm{~g} \mathrm{dl}^{-1}$ were managed by reducing the dose of ribavirin in $200 \mathrm{mg}$ increments. Patients that developed neutropenia defined as absolute neutrophil count $<0.75 \times 1091^{-1}$ or thrombocytopenia defined as platelet count $<50 \mathrm{k} \mathrm{l}^{-1}$ were managed by stepwise dose reductions of CIFN to 12,9 , or $6 \mu \mathrm{g} \mathrm{day}^{-1}$.

\section{Statistical Analysis}

Chi-squared test was used to compare significant differences between study arms. Logistic regression analysis was used to determine predictors of SVR. Statistical analyses were performed using Microsoft Excel version 2007 and SPSS software version 16.0 (SPSS, Chicago, IL).

\section{Results}

Patients

A total of 64 patients were recruited for this study at nine sites in the United States. All patients received daily CIFN (15 mcg day ${ }^{-1} \mathrm{SQ}$ ) and RBV (1-1.2 $\left.\mathrm{g} \mathrm{day}^{-1} \mathrm{PO}\right)$ given for at least 52 weeks. In addition, at enrollment 33 patients were randomized into group $\mathrm{A}$, receiving 52 weeks of treatment, and 31 patients were randomized into group B, receiving 52-72 weeks of treatment depending on time to viral undetectability within the first 24 weeks.

Baseline demographic and clinical characteristics of all treatment naïve, $\mathrm{HCV}$ genotype 1 patients who received CIFN and RBV are presented in Table 1. Overall, the patients were 94\% male, 33\% African American, 69\% had high viral load (HCV RNA levels >800,000 $\mathrm{IU} \mathrm{ml}^{-1}$ ), 55\% stage 3-4 fibrosis, and mean weight was $204 \mathrm{lb}$. The large majority of patients $(80 \%)$ were from VA medical centers. Comparison of the characteristics of the randomized groups indicated that both study groups were well matched with no significant differences between groups.
Table 1 Baseline patient characteristics

\begin{tabular}{llll}
\hline Characteristic & $\begin{array}{l}\text { Treatment } \\
\text { group A }\end{array}$ & $\begin{array}{l}\text { Treatment } \\
\text { group B }\end{array}$ & Total \\
\hline Caucasian & $22 / 33(67 \%)$ & $19 / 31(61 \%)$ & $41 / 64(64 \%)$ \\
African American & $11 / 33(33 \%)$ & $10 / 31(32 \%)$ & $21 / 64(33 \%)$ \\
Other & $0 / 33(0 \%)$ & $2 / 31(7 \%)$ & $2 / 64(3 \%)$ \\
VA patients & $26 / 33(79 \%)$ & $25 / 31(81 \%)$ & $51 / 64(80 \%)$ \\
Non-VA patients & $7 / 33(21 \%)$ & $6 / 31(19 \%)$ & $13 / 64(20 \%)$ \\
Severe fibrosis & $17 / 33(52 \%)$ & $18 / 31(58 \%)$ & $35 / 64(55 \%)$ \\
$\quad$ stage III-IV) & & & \\
Mild fibrosis (stage I-II) & $16 / 33(48 \%)$ & $13 / 31(42 \%)$ & $28 / 64(45 \%)$ \\
High viral load & $24 / 33(73 \%)$ & $20 / 31(65 \%)$ & $44 / 64(69 \%)$ \\
$\quad(>800,000 \mathrm{IU} / \mathrm{ml})$ & & & \\
Low viral load & $9 / 33(27 \%)$ & $11 / 31(35 \%)$ & $20 / 64(31 \%)$ \\
$\quad(<800,000 \mathrm{IU} / \mathrm{ml})$ & & & \\
Male & $32 / 33(97 \%)$ & $28 / 31(90 \%)$ & $60 / 64(94 \%)$ \\
Female & $1 / 33(3 \%)$ & $3 / 31(10 \%)$ & $4 / 64(6 \%)$ \\
Average weight & $207 \pm 35 \mathrm{lb}$ & $202 \pm 38 \mathrm{lb}$ & $204 \pm 36 \mathrm{lb}$ \\
\hline
\end{tabular}

$V A$ Veterans Affairs

No significant $(P<0.05)$ differences between treatment arms

\section{Antiviral Efficacy}

In order to evaluate the antiviral efficacy of CIFN on the entire patient cohort a pooled analysis of patients in both groups $\mathrm{A}$ and $\mathrm{B}$ was conducted. Note that definitions of PCR negative were based on the use of a HCV quantitative test from weeks 4 to 20 and a HCV qualitative test from week 24 and after (see "Methods"). Intention to treat analysis showed that $31 \%(20 / 64)$ of patients demonstrated a rapid virologic response (RVR) at 4 weeks (Fig. 2a). In addition, $20 \%$ (13/64) were complete early virologic responders (EVR) between 8 and 12 weeks and 14\% (9/64) were late virologic responders (LVR) between 12 and 24 weeks. As depicted in Fig. 2a, 52\% of patients overall were viral negative by week $12,52 \%$ were viral negative by week 24 , and $42 \%$ were viral negative by week 52 . Overall, 66\% (42/64) achieved undetectable viral levels at some point by week 24 , but this includes nine patients that dropped out early and were later categorized as treatment failures. Overall, in intention to treat analysis the final SVR rate was 33\% (21/64) (Fig. 2a). Separately, there were no significant differences in viral negativity rates between treatment groups A $(36 \% ; 12 / 33)$ and B $(48 \%, 15 / 31)$ through 52 weeks (Fig. 2a). Similar final SVR rates were noted in treatment arm A $(33 \% ; 11 / 33)$ and treatment arm B $(32 \%$; 10/31) (Fig. 2a, b). Of note, only two patients of the eight eligible patients in treatment arm $\mathrm{B}$ received more than 52 weeks of treatment. Six of eight patients eligible for extended duration of therapy beyond 52 weeks stopped therapy due to noncompliance, intolerance of side effects and breakthrough viremia. 


\section{A}
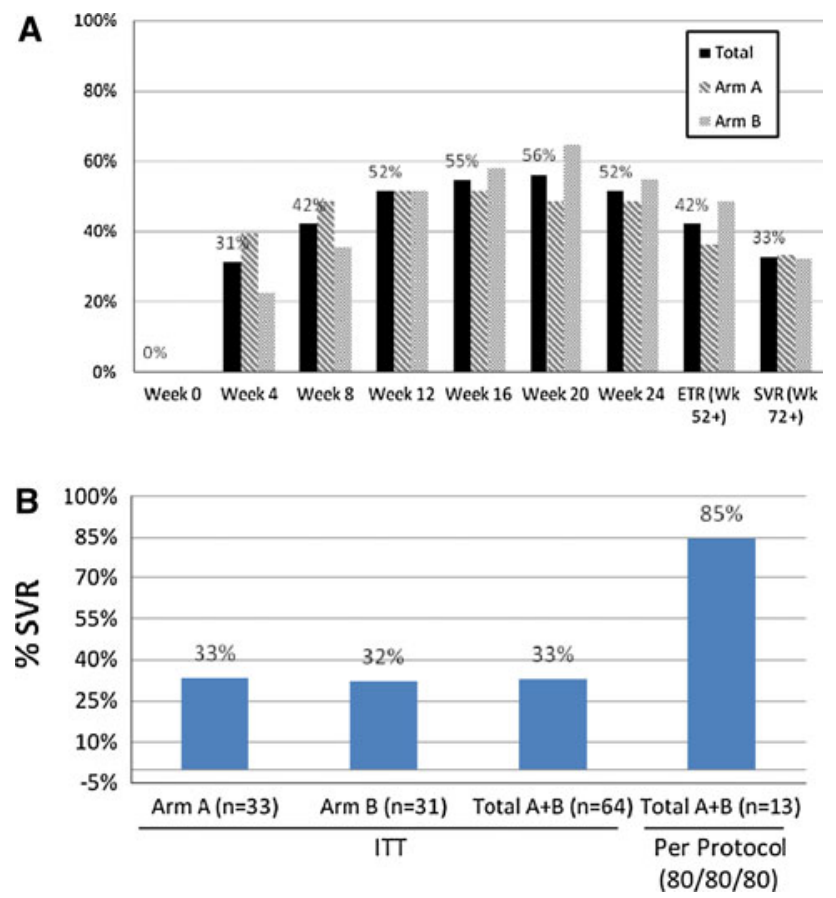

Fig. 2 Patient outcomes. a Percentage of patients with negative hepatitis $\mathrm{C}$ virus polymerase chain reaction (HCV PCR) results over time for total cohort and patients in groups $\mathrm{A}$ and $\mathrm{B}$. Results include HCV quantitative PCR negative (week 4-20) and HCV qualitative PCR negative (week 24, end of treatment [week 52+]) and end of follow up (week 72+) data. Note: patients who were HCV PCR negative but subsequently dropped out were considered as treatment failures from the time of drop out. b Sustained virologic response (SVR) analysis by study arm and overall SVR result for total cohort. Intention to treat (ITT) and overall per protocol SVR rates

Table 2 Sustained virologic response (SVR) based on patient characteristics

\begin{tabular}{llr}
\hline Group & $\begin{array}{l}\text { Number } \\
\text { of patients }\end{array}$ & \multicolumn{1}{c}{ SVR (\%) } \\
\hline RVR (week 4) & 20 & $15 / 20(75 \%)$ \\
Total EVR ( $\geq$ 2-log drop week 12) & 29 & $8 / 29(28 \%)$ \\
Partial EVR ( $\geq 2-\log$ drop but & 8 & $2 / 8(25 \%)$ \\
$\quad$ detectable week 12) & & \\
Complete EVR (PCR negative & 21 & $6 / 21(29 \%)$ \\
$\quad$ at week 12) & & $0 / 1(0 \%)$ \\
LVR (week 24) & 1 & $18 / 41(44 \%)$ \\
Caucasian & 41 & $3 / 21(14 \%)$ \\
African American & 21 & $14 / 22(32 \%)$ \\
Stage 4 fibrosis & 22 & $14 / 43(32 \%)$ \\
Stage 0-3 fibrosis & 42 & $7 / 21(33 \%)$ \\
High VL (>800 k IU/ml) & 43 & $11 / 13(85 \%)$ \\
Low VL (<800 k IU/ml) & 21 & \\
80\% Compliant $(80 / 80 / 80)$ & 13 &
\end{tabular}

$R V R$ rapid virologic response, $E V R$ early virologic responders, $P C R$ polymerase chain reaction, $L V R$ late virologic response
The relationship of virologic response during therapy and final SVR results are summarized in Table 2. Patients with RVR demonstrated a 75\% (15/20) SVR. Patients with complete EVR and LVR demonstrated 29\% (6/21) and 0\% (0/1) SVR, respectively. Per-protocol analysis including patients who received $80 \%$ of the planned CIFN and ribavirin dose through at least week 52 and who took the medication $80 \%$ of the time (80/80/80 compliance), revealed an overall SVR rate of $85 \%$ (11/13) (Table 2; Fig. 2b). Univariate analysis of possible predictors of SVR (viral load, fibrosis stage, race, RVR, and 80/80/80 compliance) indicated that patients with $80 / 80 / 80$ compliance had significantly higher SVR rates compared to those who did not $(P<0.05)$.

We noted a high degree of variance in terms of SVR rates and early discontinuation of patients from treatment within the first 24 weeks at different study sites. Figure 3 illustrates four centers with the greatest numbers of patients, and compares the percent of patients with early virologic response, SVR, and early discontinuation within the first 24 weeks. These data indicate a trend toward an inverse correlation between SVR rate and early discontinuation rates. Although this analysis is limited by low numbers of patients and variable numbers of African American patients at each site, these data suggest the importance of each site in maintaining patient adherence to therapy and final SVR rate.

\section{Safety and Tolerability}

There was an increased rate of dose reduction and discontinuation before the recommended duration of treatment (Table 3). Overall, during treatment $61 \%$ (39/64) of patients required CIFN dose reduction and 41\% (26/64) required RBV dose reduction. The timing and reasons for CIFN and RBV dose reduction are indicated in Fig. 4a and $b$. Dose reductions tended to occur early during the

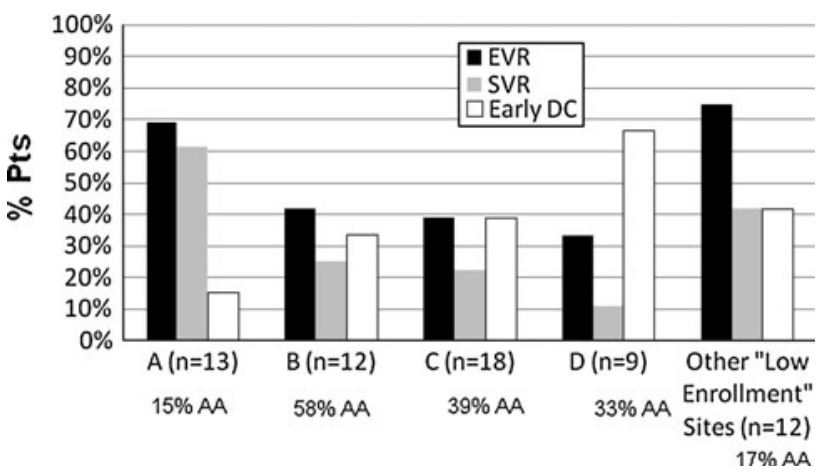

Fig. 3 Patient outcomes by study site. Sites with greater than eight patients enrolled were compared (sites $A-D$ ), and other study sites with lower enrollment were grouped together (other low enrollment sites). AA African American 
Table 3 Safety analysis

\begin{tabular}{|c|c|c|c|}
\hline Treatment discontinuations & $\begin{array}{l}\text { Group A } \\
(N=33)\end{array}$ & $\begin{array}{l}\text { Group B } \\
(N=31)\end{array}$ & $\begin{array}{l}\text { Total } \\
(N=64)\end{array}$ \\
\hline Serious adverse events ${ }^{\mathrm{a}}$ & $1(3.2 \%)$ & $1(3.2 \%)$ & $2(3.1 \%)$ \\
\hline $\begin{array}{l}\text { Discontinuations due to } \\
\text { adverse events or } \\
\text { noncompliance }\end{array}$ & $22(66.7 \%)$ & $10(32.2 \%)^{\mathrm{b}}$ & $32(50.0 \%)$ \\
\hline $\begin{array}{l}\text { Discontinuations due to } \\
\text { virologic non-efficacy }\end{array}$ & $2(6.1 \%)$ & $8(25.8 \%)^{\mathrm{c}}$ & $10(15.6 \%)$ \\
\hline $\begin{array}{l}\text { Patients requiring } \\
\text { erythropoetin }\end{array}$ & $4(12.1 \%)$ & $3(9.7 \%)$ & $7(10.9 \%)$ \\
\hline Patients requiring G-CSF & 0 & $5(16.1 \%)^{\mathrm{d}}$ & $5(7.8 \%)$ \\
\hline
\end{tabular}

${ }^{a}$ SAEs that resulted in hospitalization included chest pain and encephalopathy

${ }^{\text {b } P=0.01}$

c $P=0.04$ (total discontinuations for any reason $P=0.29$ )

${ }^{\mathrm{d}} P=0.02$
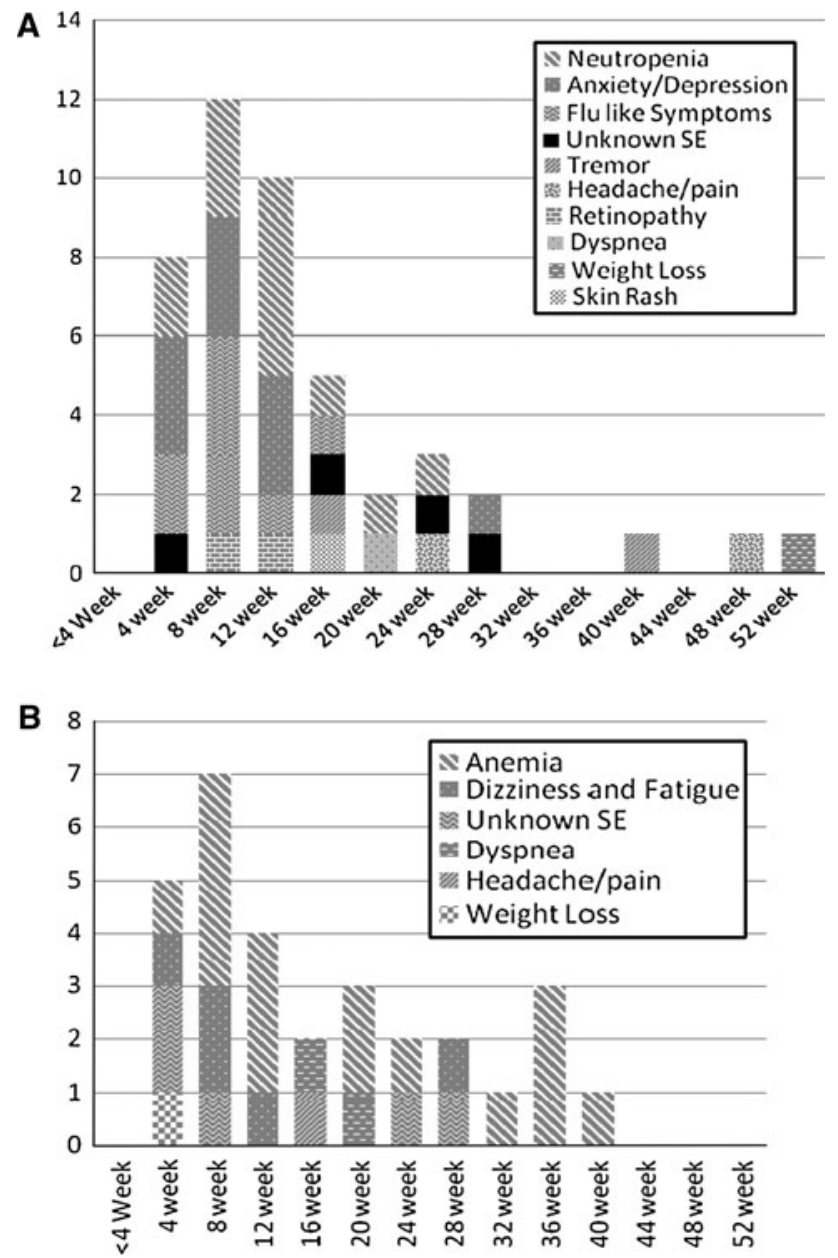

Fig. 4 Dose reductions. a Number and reasons for incidences of consensus interferon (CIFN) dose reduction. b Number and reasons for incidences of ribavirin (RBV) dose reduction

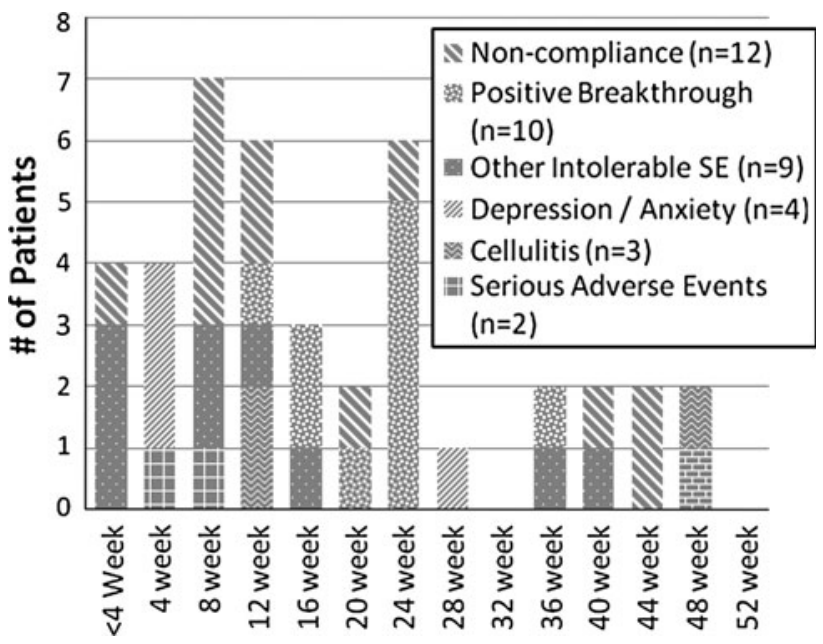

Fig. 5 Number and reasons for incidences of treatment discontinuation

course of treatment. Early discontinuation of treatment before 24 weeks occurred in $40 \%$ (26/64) of patients (12 due to intolerance, 11 due to noncompliance, 1 due to chest pain, and 2 due to cellulitis), as indicated in Fig. 5. Treatment discontinuations due to serious adverse events occurred in two patients, which were hospitalizations due to chest pain and encephalopathy, respectively. Overall, discontinuations due to adverse events or noncompliance occurred in $50 \%$ of patients, with the greatest number of discontinuation occurring in the first 12 weeks. Discontinuation due to virologic non-efficacy occurred in $15.6 \%$. As indicated in Table 3, significant differences were observed between groups A and B in numbers of patients with discontinuations for "Discontinuations due to adverse events or noncompliance" and "Discontinuations due to virologic non-efficacy", but overall there was no difference in overall discontinuations between the two groups $(P=0.29)$. The reason for the differences between groups in these two types of discontinuations is unknown, as they occurred in both groups before the 52-week point when there was no difference in treatment and can only be attributed to the small sample size. In addition there was a significant difference in "Patients requiring G-CSF", but again this is unexplained and likely due to the small sample size.

\section{Discussion}

The purpose of this study was to determine the efficacy and safety of a regimen of daily CIFN and ribavirin in HCV genotype 1 patients with "difficult to treat" characteristics. This multicenter study enrolled patients that were 94\% male and $33 \%$ African American, with $80 \%$ of patients at VA medical centers. In addition, the majority of these patients had advanced fibrosis and a high viral load. On an 
intention to treat basis we found that $31 \%$ achieved a rapid virologic response and an overall $33 \%$ SVR rate. This trial failed to test the difference between 52 weeks of treatment vs. variable treatment duration of 52-72 weeks, due to the fact that so few patients required or were able to comply with therapy beyond 52 weeks of treatment. Overall, we observed frequent early discontinuation of therapy due to noncompliance or side effects; however, there was a wide variation in patient compliance between sites. Patients that adhered with at least $80 \%$ of the prescribed dose of CIFN and ribavirin for $80 \%$ of the prescribed duration to 52 weeks achieved an $85 \%$ SVR rate.

Prior clinical studies of CIFN and ribavirin have indicated mixed results. Pockros et al. reported 40 treatment naïve patients in community/academic practices, $50-55 \%$ genotype 1 , who received either CIFN $9 \mu \mathrm{g} /$ day with ribavirin 1,000-1,200 mg/d or CIFN $9 \mu \mathrm{g} / \mathrm{d}$ alone [21]. They found that $65 \%$ of patients who received CIFN and ribavirin required dose reductions, with an overall $35 \%$ discontinuation rate. The overall SVR rate in patients receiving CIFN and ribavirin was $40 \%$. On the other hand, several clinical studies reported greater antiviral responses with CIFN in patients with genotype 1 hepatitis $\mathrm{C}$ infection, particularly if given as a daily injection. Recent data indicate that $15 \mu \mathrm{g}$ CIFN given three times a week with ribavirin has an equivalent SVR rate to pegylated interferon alfa-2a and ribavirin in patients with $\mathrm{HCV}$ genotype 1 [16].

Preliminary data have also indicated that up to $39-47 \%$ $\mathrm{HCV}$ genotype 1 patients who do not respond to interferon alfa-2b + ribavirin may respond to daily induction dosing of CIFN (18 or $27 \mu \mathrm{g} / \mathrm{d}$ for 4 weeks followed by 9 or $18 \mu \mathrm{g} / \mathrm{d}$ for 8 weeks, then $9 \mu \mathrm{g} / \mathrm{d}$ for 36 weeks) with the addition of ribavirin and have a sustained virologic response [14]. Furthermore, Kaiser et al. [15] have shown that daily high dose induction dosing of CIFN with ribavirin therapy results in sustained response rates in about one quarter of previous pegylated interferon and ribavirin non-responders. Kaiser et al. have also published preliminary data treating treatment-naive genotype 1 patients in Germany with daily high doses CIFN and ribavirin for 48 weeks. They found that $59-67 \%$ of genotype 1 patients experienced a sustained virologic response [14]. Leevy et al. [17] recently reported that U.S. patient nonresponders to pegylated interferon and ribavirin who receive daily CIFN (15 $\mu \mathrm{g})$ and ribavirin can achieve a $37 \%$ sustained virologic response rate. Bacon et al. [18] in the multicenter DIRECT trial treated patients who were prior pegylated interferon and ribavirin nonresponders with CIFN ( 9 or $15 \mu \mathrm{g} / \mathrm{d}$ ) and ribavirin and found an overall 6.9-10.7\% $\mathrm{SVR}$ rate. In two relapse studies, CIFN was compared to pegylated interferon showing greater efficacy at either 15 or $9 \mu \mathrm{g} / \mathrm{d}$ ( $69 \%$ vs. $42 \%$ and $47 \%$ vs. $29 \%$, respectively)
[22, 23]. However, to date there have been no published direct comparisons of daily CIFN and ribavirin with pegylated interferon and ribavirin for HCV treatment naïve or nonresponder patients. Given the mixed results, our data emphasizes the difficulties in tolerance of this regimen, and adequate patient support is essential and may explain the differences reported between centers.

The overall $33 \%$ SVR rate obtained in the genotype 1 patients in this study compares favorably with the few prior reports of the efficacy of pegylated interferon and ribavirin for male predominant VA patients with HCV genotype 1 . Backus et al. [9] reported an overall 20\% SVR rate for $\mathrm{HCV}$ genotype 1 patients who received pegylated interferon and ribavirin in the national VA HCV registry database. Single VA centers have reported SVR rates of $15-33 \%$ in smaller numbers of patients [24-26].

Patients treated with daily CIFN and ribavirin demonstrated a $31 \%$ rapid virologic response rate, defined as a HCV PCR test below the limit of detection on a quantitative PCR assay at 4 weeks. One limitation of this study was the use of a quantitative PCR test during weeks 4-20, with lower limits of detection from 100 to $600 \mathrm{IU} \mathrm{ml}^{-1}$. In addition, the methods used for HCV PCR tests varied from site to site. Therefore the data presented may overestimate the true viral negative rate because of the sites using an assay with a lower limit of detection of $600 \mathrm{IU} \mathrm{ml}^{-1}$. If we limited the analysis to sites that used a quantitative assay with a lower limit of detection of $50 \mathrm{IU} \mathrm{ml}^{-1}$ we observe that $12 / 51(23.5 \%)$ patients were HCV PCR negative at week 4 and therefore have a rapid virologic response. This can be compared with another U.S. trial of genotype 1 treatment naïve patients treated with either pegylated interferon alfa- $2 \mathrm{a}$ or alfa-2b and ribavirin, which demonstrated a $11.4-11.9 \%$ rapid virologic response rate [7]. In contrast, recent European trials examining the relevance of extended therapy based on virologic response in patients with $\mathrm{HCV}$ genotype 1 treated with pegylated interferon alfa and ribavirin used HCV PCR assays with a lower limit of detection of $50 \mathrm{IU} \mathrm{ml}^{-1}$ to determine virologic response at 4 weeks [19, 20]. These studies found that 19-22\% of these patients had a rapid virologic response. In addition, it remains unknown how CIFN will be used in the upcoming era of targeted therapy, but treatment strategies associated with a high initial RVR may be important when considering the addition of more specific antivirals in order to reduce the chance of viral mutations and for determination of duration of therapy [27].

Patients in this trial were observed to have a relative high early discontinuation rate of $40 \%$ within the first 24 weeks and a $50 \%$ overall discontinuation rate due to adverse events and noncompliance. This is only somewhat higher than previous early discontinuation rates published for comparable male predominant VA type patient 
populations. For example, Brau et al. [28] reported a 30.5\% discontinuation rate for adverse events during treatment in a 785 patient VA cohort treated with standard interferon alfa and ribavirin. Backus et al. [9] reported that $62 \%$ of patients received less than the targeted full treatment duration in a retrospective analysis of 5,944 patients in the VA Clinical Case Registry for hepatitis C treated with pegylated interferon and ribavirin. Note that this includes patients that were discontinued for any reason, including lack of efficacy in addition to presumed adverse events, and the number of patients that discontinued within the first 24 weeks of treatment was not reported. In contrast, studies from registration trials of pegylated interferon and ribavirin reported discontinuation rates for adverse events of 14.0-22\% [4, 5, 29]. In addition, Bacon et al. [18] reported a $21 \%$ discontinuation rate due to adverse events in the DIRECT registration trial patients treated with daily CIFN and ribavirin using dosing similar to the present study. Note that this trial included only patients with previous treatment experience, who were nonresponders to prior treatment with pegylated interferon and ribavirin and motivated to receive repeat treatment. These data indicate that treatment discontinuation reflects the patient population in addition to the type of regimen used. Furthermore, we observed that compliance with treatment and discontinuation rates correlated with study site, which may be another explanation for the relatively overall high discontinuation rate observed in this study. These data emphasize the importance of supportive care for continuing patients on daily CIFN and ribavirin treatment. Overall we found that daily CIFN and ribavirin was safe in these patients, with only $2(3.1 \%)$ serious adverse events reported, which is comparable with the rate of serious adverse events in a recent U.S. trial of pegylated interferon alfa- $2 a$ and alfa- $2 b$ with ribavirin [7].

In conclusion, daily CIFN and ribavirin for initial treatment of $\mathrm{HCV}-1$ patients with a high incidence of difficult-to-treat characteristics results has potential for achieving a relatively high rapid virologic response rate, but discontinuations are frequent and successful use of this regimen is highly dependent on adequate patient support to maintain adherence.

Acknowledgments The authors would like to acknowledge the assistance of Dr. Anastasios A. Mihas of the Hunter Holmes McGuire VA Medical Center, Richmond, VA, USA, and the Research Service of the VA San Diego Healthcare System.

Authors' declaration of personal interests Samuel B. Ho, MD, has served as a speaker for Three Rivers Pharmaceuticals, and has received research funding from Three Rivers Pharmaceuticals, Valeant Pharmaceuticals International, and Human Genome Systems. Bashar Aqel,Eric Dieperink,Shanglei Liu and Lori Tetrick have no disclosures. Yngve Falck-Ytter: Research support and speaker's honorarium from Roche Pharmaceuticals. Coleman Smith: Research support and speaker's honorarium from Bristol-Myers Squibb, Novartis Pharmaceuticals, Schering-Plough Corporation, Gilead, and Roche Pharmaceuticals. Edmund J. Bini: Research support and speaker's from Bristol-Myers Squibb, Pharmasset, Schering-Plough Corporation, Gilead, Roche Pharmaceuticals, Valeant, Vertex. Marcos C. Pedrosa: Research support and speaker's honorarium from Schering-Plough Corporation.

Declaration of funding interests This study was funded by Three Rivers Pharmaceuticals and Valeant Pharmaceuticals International and supported by the Veterans Affairs Research Service.

Open Access This article is distributed under the terms of the Creative Commons Attribution Noncommercial License which permits any noncommercial use, distribution, and reproduction in any medium, provided the original author(s) and source are credited.

\section{References}

1. Armstrong GL, Wasley A, Simard EP, et al. The prevalence of hepatitis C virus infection in the United States, 1999 through 2002. Ann Intern Med. 2006;144:705-714.

2. Wong JB, McQuillan GM, McHutchison JG, et al. Estimating future hepatitis $\mathrm{C}$ morbidity, mortality, and costs in the United States. Am J Public Health. 2000;90:1562-1569.

3. NIH. National Institutes of Health consensus development conference statement: management of hepatitis C: 2002. Hepatology. 2002;36:S3-S15.

4. Manns MP, McHutchison JG, Gordon SC, et al. Peginterferon alfa- $2 b$ plus ribavirin compared with interferon alfa- $2 b$ plus ribavirin for initial treatment of chronic hepatitis $\mathrm{C}$ : a randomised trial. Lancet. 2001;358:958-965.

5. Fried MW, Shiffman ML, Reddy KR, et al. Peginterferon alfa-2a plus ribavirin for chronic hepatitis $\mathrm{C}$ virus infection. $N$ Engl $J$ Med. 2002;347:975-982.

6. Jacobson IM, Brown RS Jr, Freilich B, et al. Peginterferon alfa$2 \mathrm{~b}$ and weight-based or flat-dose ribavirin in chronic hepatitis $\mathrm{C}$ patients: a randomized trial. Hepatology. 2007;46:971-981.

7. McHutchison JG, Lawitz EJ, Shiffman ML, et al. Peginterferon Alfa-2b or Alfa-2a with ribavirin for treatment of hepatitis C infection. N Engl J Med. 2009;361:580-593.

8. Bini EJ, Brau N, Currie S, et al. Prospective multicenter study of eligibility for antiviral therapy among 4,084 U.S. Veterans with chronic hepatitis C virus infection. Am J Gastroenterol. 2005; 100:1772-1779.

9. Backus LI, Boothroyd DB, Phillips BR, et al. Predictors of response of US veterans to treatment for the hepatitis $\mathrm{C}$ virus. Hepatology. 2007;46:37-47.

10. Blatt LM, Davis JM, Klein SB, et al. The biologic activity and molecular characterization of a novel synthetic interferon-alpha species, consensus interferon. J Interferon Cytokine Res. 1996; 16:489-499.

11. Ozes ON, Reiter Z, Klein S, et al. A comparison of interferonCon1 with natural recombinant interferons-alpha: antiviral, antiproliferative, and natural killer-inducing activities. J Interferon Res. 1992;12:55-59.

12. Klein SB, Blatt LM, Taylor MW. Consensus interferon induces peak mRNA accumulation at lower concentrations than interferon-alpha 2a. J Interferon Res. 1993;13:341-347.

13. Klein SB, Blatt LM, Taylor MW. Cell surface binding characteristics correlate with consensus type I interferon enhanced activity. J Interferon Cytokine Res. 1996;16:1-6. 
14. Kaiser S, Hass H, Gregor M. High dose induction therapy with consensus interferon and ribavirin for treatment-naive patients with hepatitis C. Hepatology. 2002;36:362A.

15. Kaiser S, Hass H, Gregor M. Successful retreatment of peginterferon nonresponder patients with chronic hepatitis $\mathrm{C}$ with high dose consensus interferon induction therapy. Gastroenterology. 2004;126:A668.

16. Sjogren MH, Sjogren R Jr, Lyons MF, et al. Antiviral response of HCV genotype 1 to consensus interferon and ribavirin versus pegylated interferon and ribavirin. Dig Dis Sci. 2007;52: $1540-1547$.

17. Leevy CB. Consensus interferon and ribavirin in patients with chronic hepatitis $\mathrm{C}$ who were nonresponders to pegylated interferon alfa-2b and ribavirin. Dig Dis Sci. 2008;53:1961-1966.

18. Bacon BR, Shiffman ML, Mendes F, et al. Retreating chronic hepatitis $\mathrm{C}$ with daily interferon alfacon-1/ribavirin after nonresponse to pegylated interferon/ribavirin: DIRECT results. Hepatology. 2009;49:1838-1846.

19. Berg T, von Wagner M, Nasser S, et al. Extended treatment duration for hepatitis $\mathrm{C}$ virus type 1: comparing 48 versus 72 weeks of peginterferon-alfa-2a plus ribavirin. Gastroenterology. 2006;130:1086-1097.

20. Sanchez-Tapias JM, Diago M, Escartin P, et al. Peginterferonalfa2a plus ribavirin for 48 versus 72 weeks in patients with detectable hepatitis C virus RNA at week 4 of treatment. Gastroenterology. 2006;131:451-460.

21. Pockros PJ, Reindollar R, McHutchinson J, et al. The safety and tolerability of daily infergen plus ribavirin in the treatment of naive chronic hepatitis C patients. J Viral Hepat. 2003;10:55-60.

22. Kaiser S, Lutze B, Sauter B, et al. Retreatment of HCV genotype 1 relapse patients to peginterferon/ribavirin therapy with an extended treatment regimen of 72 weeks with consensus interferon/ribavirin versus peginterferon alpha/ribavirin. Hepatology. 2007;46:819A.

23. Pearlman B, Ehleben C. Retreatment of chronic hepatitis C genotype 1 infected relapsers to peginterferon/ribavirin with consensus interferon/ribavirin or with extended duration therapy peginterferon/ribavirin. Hepatology. 2009;50:686A.

24. Chainuvati S, Khalid SK, Kancir S, et al. Comparison of hepatitis $\mathrm{C}$ treatment patterns in patients with and without psychiatric and/ or substance use disorders. J Viral Hepat. 2006;13:235-241.

25. Knott A, Dieperink E, Willenbring ML, et al. Integrated psychiatric/medical care in a chronic hepatitis $\mathrm{C}$ clinic: effect on antiviral treatment evaluation and outcomes. Am J Gastroenterol. 2006;101:2254-2262.

26. Groom H, Dieperink E, Nelson DB, et al. Outcomes of a hepatitis C screening program at a large urban VA Medical Center. J Clin Gastroenterol. 2008;42:97-106.

27. Kwo PY, Lawitz EJ, McCone J, et al. Efficacy of boceprevir, an NS3 protease inhibitor, in combination with peginterferon alfa- $2 b$ and ribavirin in treatment-naive patients with genotype 1 hepatitis C infection (SPRINT-1): an open-label, randomised, multicentre phase 2 trial. Lancet. 2010;376:705-716.

28. Brau N, Bini EJ, Currie S, et al. Black patients with chronic hepatitis $\mathrm{C}$ have a lower sustained viral response rate than nonBlacks with genotype 1 , but the same with genotypes $2 / 3$, and this is not explained by more frequent dose reductions of interferon and ribavirin. $J$ Viral Hepat. 2006;13:242-249.

29. Hadziyannis SJ, Sette H Jr, Morgan TR, et al. Peginterferonalpha2a and ribavirin combination therapy in chronic hepatitis $\mathrm{C}$ : a randomized study of treatment duration and ribavirin dose. Ann Intern Med. 2004;140:346-355. 